and bacterial challenge at baseline, and following $2 \mathrm{~h}$ stimulation with lipopolysaccharide (LPS) and ammonia. Pro- and anti-inflammatory cytokines were determined by CBA.

Results Baseline neutrophil TLR9 expression was significantly higher in patients with HE (ALF: Grade 3/4 vs controls: $p<0.02$, vs grade $0-2: p<0.03$ ) (Cirrhotics: Grade $3 / 4$ vs controls: $p<0.03$, vs grade $0-2: p<0.05)$. Moreover their baseline TLR9 expression was associated with severity of HE and higher IL6 and IL8 levels. CD16 expression was downregulated by a median of $45 \%$ (range $25 \%-$ $85 \%$ ) in ALF patients with grade $3 / 4 \mathrm{HE}$ compared to controls and in cirrhotics by a median of $88 \%$ (range $5 \%-90 \%$ ) (Grade $3 / 4$ vs controls: $\mathrm{p}<0.05$ ). Baseline CD11b expression did not differ between controls and patients. Exposure to LPS and ammonia upregulated TLR9 and CD11b and downregulated CD16.

Conclusion Neutrophil TLR9 expression in patients with ALF and cirrhosis serves as a useful biomarker that differentiates those who develop high grade HE from those who do not. High baseline TLR9 expression and low CD16 expression promote a pro-inflammatory cytokine milieu that may help to explain the propensity to develop infection and why inflammation hastens the development of HE. TLR9 antagonists may be of therapeutic value in restoring neutrophil activity.

Competing interests None declared.

\section{PM0-126 THE ROLE OF VASCULAR-ADHESION-PROTEIN 1 (VAP-1) IN MEDIATING MONOCYTE MIGRATION ACROSS INFLAMED HEPATIC SINUSOIDAL ENDOTHELIUM}

doi:10.1136/gutjnl-2012-302514b.126

H Zimmermann,* C J Weston, S M Curbishley, D H Adams. NIHR Biomedical Research Unit and Centre for Liver Research, University of Birmingham, Birmingham, UK

Introduction There is compelling evidence that accumulating monocyte-derived macrophages are pivotally involved in driving liver fibrogenesis. It remains unclear which molecules mediate transmigration of these cells across hepatic sinusoidal endothelial cells (HSEC). VAP-1 is an atypical adhesion molecule with enzymatic monoamine oxidase activity that is predominantly expressed in the liver microvasculature. It possesses key function in the recruitment of various lymphocyte subsets. The aim of this study was to decipher VAP-1 contribution to monocytic transendothelial transmigration.

Methods Primary human HSEC were isolated from explanted and grown to confluence in flow chambers. After activation with TNF$\alpha /$ IFN- $\gamma$ for $24 \mathrm{~h}$ HSEC were treated with VAP-1 antibody and enzyme inhibitors. Monocytes were enriched from peripheral blood by using OptiPrep gradient. Monocyte subsets (CD14 ${ }^{++} \mathrm{CD} 16^{-}$, $\left.\mathrm{CD} 14^{++} \mathrm{CD} 16^{+}, \mathrm{CD} 14^{+} \mathrm{CD} 16^{++}\right)$were isolated by FACS-sorting. Isolated monocytes were perfused over HSEC monolayers under constant flow simulating physiological shear stress $(0.05 \mathrm{~Pa})$. Adhesion and transmigration was studied using phase contrast microscopy. Transwell assays were used to study the phenotype of transmigrated monocytes by flowcytometry.

Results HSEC pretreatment with VAP-1 antibody (TK8-14) or enzyme inhibitor Semicarbizide equally reduced monocyte transmigration by $\sim 50 \%$. VCAM-1 blockade had a similar but redundant effect whereas CLEVER-1-antibody or LOX-inhibitor ( $\beta$-APN) did not alter monocyte transmigration. VAP-1 antibody acted in a timedependent manner with influence on monocyte adhesion only after short-term application (15 min). Inhibiting VAP-1 led to profound reduction of proinflammatory nonclassical $\mathrm{CD} 14^{+} \mathrm{CD} 16^{++}$monocyte transmigration but also affected classical $\mathrm{CD} 14^{++} \mathrm{CD} 16^{-}$ whereas the intermediate $\mathrm{CD} 14^{++} \mathrm{CD} 16^{+}$subtype was not affected. Under static conditions VAP-1 enzymatic or antibody inhibition was significantly blunted suggesting flow to be a mandatory prerequisite for the biological function of VAP-1 on monocytes. Increased expression of HLA-DR and the M2 macrophage marker CD206 on monocyte subsets after endothelial transmigration was not altered by VAP-1 inhibition.

Conclusion Endothelial VAP-1 differentially modulates monocyte recruitment under flow conditions in a time-dependent fashion and . favours transmigration of a proinflammatory monocyte subset. The critical role of VAP-1 enzyme function renders small molecules as a promising therapeutic approach in combating liver inflammation and subsequent fibrosis.

Competing interests None declared.

\section{PMO-127 BIOLOGICAL EFFECTS OF ORAL NANOPOROUS CARBON IN BILE DUCT LIGATED RATS}

doi:10.1136/gutjnl-2012-302514b.127

1J Macnaughtan,* $1 \mathrm{~J}$ Soeda, ${ }^{1} \mathrm{~A}$ Mouralidarane, ${ }^{2} \mathrm{~S}$ Sandeman, ${ }^{2} \mathrm{C}$ Howell, ${ }^{3} \mathrm{O}$ Kozynchenko, ${ }^{3} \mathrm{~S}$ Tennison, ${ }^{2} \mathrm{~S}$ Mikhalovsky, ${ }^{1} \mathrm{~N}$ Davies, ${ }^{1} \mathrm{~J}$ Oben, ${ }^{1} \mathrm{R}$ Mookerjee, ${ }^{1} \mathrm{R}$ Jalan. ${ }^{1}$ Hepatology, UCL, London, UK; ${ }^{2}$ Centre for Biomedical and Health Science Research, University of Brighton, Brighton, UK; ${ }^{3}$ Mast Carbon, Guildford, UK

Introduction Gut-derived bacterial products and associated dysregulated inflammatory response play a central role in the pathogenesis of cirrhosis. Therapeutic options to target these factors are currently limited to long-term antibiotics. Nanoporous carbons are nonabsorbable, synthetic materials which are safe with porosity manipulated for adsorption of middle and high molecular weight molecules and surface chemistry modified to alter adsorption capacity for biological molecules such as cytokines and endotoxin. We sought to determine their biological effects in bile-duct ligated rats as a model of cirrhosis.

Methods 131 male Sprague-Dawley rats underwent bile duct-ligation or sham biliary surgery. Animals were pair fed with or without oral carbon therapy 2 weeks from surgery until completion of the experiment at 4-5 weeks. Intra-peritoneal lipopolysaccharide (LPS) was administered to four subgroups $3.5 \mathrm{~h}$ prior to completion of the study. The following groups were studied: Sham $(n=16)$, Sham + carbon $(n=15)$, Sham + LPS $(n=11)$, Sham + LPS + carbon $(n=10)$, BDL $(n=27), B D L+$ carbon $(n=26), B D L+L P S(n=10), B D L+L P S$ + carbon $(n=16)$. Portal haemodynamics were performed on 93 rats and Kupffer cell (KC) numbers and Reactive oxygen species (ROS) production assessed by flow cytometry in a sub-group of animals. Liver biochemistry and portal venous cytokines were performed.

Results A significant reduction in portal pressure was observed in BDL+LPS (mean 18.05 $\pm 0.88 \mathrm{~mm} \mathrm{Hg}$ untreated, $10.17 \pm 1.07 \mathrm{~mm}$ $\mathrm{Hg}$ with carbon, $\mathrm{p}<0.05$ ) and $\mathrm{BDL}$ (mean $12.57 \pm 0.43 \mathrm{~mm} \mathrm{Hg}$ untreated, $11.02 \pm 0.28 \mathrm{~mm} \mathrm{Hg}$ with carbon, $\mathrm{p}<0.05$ ) groups following carbon treatment. A significant reduction in ALT was observed in the carbon treated BDL+LPS $(p<0.05)$ and BDL groups $(p<0.05)$. Carbon treatment in BDL rats was associated with a significant reduction in LPS-induced ROS production. A trend towards reduction in portal venous IL-4 and IL-10 was observed in carbon-treated BDL rats. No significant difference in portal venous TNF- $\alpha$ was observed. Finally, a significant increase in body mass was observed in the BDL carbon-treated group $(\mathrm{p}<0.05)$.

Conclusion Oral nanoporous carbon therapy results in a significant reduction in portal pressure and liver biochemistry associated with a reduction in endotoxin-induced KC ROS production. We postulate therefore, that the effect of nanoporous carbon is possibly via a reduction in endotoxin induced $\mathrm{KC}$ stimulation.

Competing interests None declared. 